\title{
Analysis of Influence of Tilt Angle on Variable-Speed Fixed- Pitch Floating Offshore Wind Turbines for Optimizing Power Coefficient Using Experimental and CFD Models
}

\author{
Wongsakorn Wisatesajja, Wirachai Roynarin*, Decha Intholo
}

Engineering Faculty, Rajamangala University of Technology Thanyaburi (RMUTT), Pathum Thani 12110, Thailand

\begin{abstract}
This study focused on optimization of the power coefficient of floating offshore wind turbines (FOWTs) to maintain their wind power performance in order to overcome problems with the tilt angle resulting from an unstable wind turbine platform, which can reduce the effective area of wind turbine energy extraction. FOWTs with a variable-speed fixed-pitch control strategy were investigated using an experimental model in a wind tunnel and a CFD simulation model for analysis and comparison, using wind speeds of $2-5.5 \mathrm{~m} / \mathrm{s}$ and tilt angles of $3.5-6.1^{\circ}$. The results showed that average rotational speed differences of $16.4 \%$ and optimal power coefficients of $0.35-$ 0.36 could be maintained at tip speed ratios of 7.7-9.6 during wind speeds of $3-5 \mathrm{~m} / \mathrm{s}$ with tilt angles of 3.9-5.8 $8^{\circ}$. The results of this study provide insights into a new concept of power coefficient optimization using variable tilt angle for small to medium fixed pitch FOWTs, to reduce the cost of pitch control systems.
\end{abstract}

Keywords: computational fluid dynamics, floating offshore wind turbines, power coefficient, tilt angle, wind energy

Article History: Received: 29th Sept 2020; Revised: 24th Nov 2020; Accepted: $1^{\text {st }}$ Dec 2020; Available online: $4^{\text {th }}$ Dec 2020

How to Cite This Article: Wisatesajja, W., Roynarin, W., Intholo, D. (2021) Analysis of Influence of Tilt Angle on Variable-Speed Fixed-Pitch Floating Offshore Wind Turbines for Optimizing Power Coefficient Using Experimental and CFD Models. Int. Journal of Renewable Energy Development, 10(2), 201-212

https://doi.org/10.14710/ijred.2021.33195

\section{Introduction}

For several years, renewable energy has been considered to be an alternative source of electricity apart from fossil fuels, in terms of economic and environmental preservation. Recently, power plants operating using solar photovoltaic (PV) systems and wind have been shown to produce electricity more cheaply than fossil fuels can, in many locations. It has been estimated that more than $26 \%$ of renewable energy could be used to produce electricity globally, which could lead to a greater renewable energy capacity than is possible with fossil fuels and nuclear power combined (REN21 2019). Floating offshore wind turbines (FOWTs) have been developed to address the weaknesses of onshore wind turbines, such as wind speed problems, installation site issues, and visual issues (REN21 2018). According to the 2019 International Renewable Energy Agency (IRENA) Renewable Capacity Statistics and as reported in 2018, the total global onshore wind turbine capacity was 539,954 MW, while the total global offshore wind turbine capacity was 23,706 MW (International Renewable Energy Agency 2019).

Thailand is geographically situated in a low-wind-speed area with average wind speed of $4-4.5 \mathrm{~m} / \mathrm{s}$. Therefore, a low-wind-speed airfoil blade called R1235 was developed specifically to operate in low-wind-speed energy resource areas. The first $205-\mathrm{kW}$ wind turbines, equipped with R1235 airfoil blades, were installed at Phetchaburi Province as part of the King Rama 9 project; the total capacity of the turbines was $100 \mathrm{~kW}$. A second set of wind turbines with R1235 airfoil blades and a capacity of $10 \mathrm{~kW}$ was installed at Chonburi Province as part of the Laem Chabang Green Port project; the total capacity was $840 \mathrm{~kW}$. Finally, a $100-\mathrm{kW}$ wind turbine project was undertaken by MRP Engineering Company at Chonburi Province to supply electricity for industrial purposes. In these three projects, the wind turbines provided a high power coefficient of 0.35 . However, the overall performance or plant factor decreased due to obstructions such as buildings, trees, and mountains surrounding the wind turbine site. These obstructions produced turbulence and wind fluctuations, which reduced the plant factor. Therefore, a FOWT was introduced to improve the wind turbine performance and plant factor of the R1235 airfoil blades. The offshore location involves less turbulence, with continuous wind flows, and the turbine is the first FOWT prototype in Thailand.

The tilt angle of a wind turbine rotor blade results from wind flow, which causes the rotor blade on a turbine to skew

\footnotetext{
* Corresponding author: wirachai_r@rmutt.ac.th
} 
from its original position, thus reducing the effective area of the rotor blade. This leads to reduced wind energy conversion (Ponta et al. 2016; 5. Gumula et al. 2017). This study examined the optimal power coefficient with respect to the influence of the tilt angle of variable-speed fixedpitch blades on FOWTs. The results were obtained using an experimental model in a wind tunnel and a computational fluid dynamics (CFD) simulation program called CFDesign v7. In addition, analyses and comparisons were performed for wind speeds of $2-5.5 \mathrm{~m} / \mathrm{s}$ to assess various aspects of wind turbine performance, including the rotational speed, tilt angle, tip speed ratio, and power coefficient.

\section{Theory}

\subsection{Wind Energy Resources}

Wind is created by air moving from high-pressure areas to low-pressure areas, as well as temperature differences resulting from the Earth's surface absorbing solar radiation. Electricity can be produced by converting wind power into electrical power using a wind turbine. Rotor blades on wind turbines rotate as wind flows, driving a generator that creates electricity. The best wind farm sites are located in coastal areas, at the tops of rounded hills, in open areas, and in the ocean (Lehr, Keeley, and Kingery 2016).

Wind energy is beneficial in several environmental, economic, and social respects. In modern society, electricity is essential for daily life. Using wind energy to produce electricity reduces the consumption of fossil fuels, decreases carbon dioxide emissions, and is an environmentally friendly and sustainable practice that can conserve energy resources for future generations (Kondili and Kaldellis 2012). In addition, economic growth can be stimulated by wind energy markets. Development of wind energy enables the creation of new jobs in manufacturing, installation, and maintenance of wind turbines, as well as consulting, operational, legal, and transportation services. Regional development, including improvements to infrastructure (roads, power grids, and transportation) and local economies, is also stimulated during the operational and maintenance phases of wind energy utilization (U.S. Department of Energy 2015).

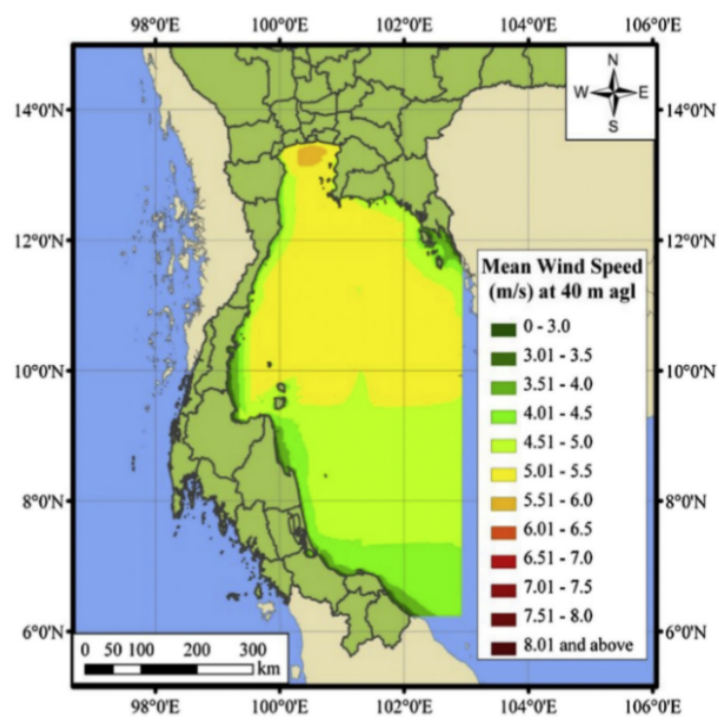

Fig. 1 Mean wind speed at $40 \mathrm{~m}$ above sea level in the Gulf of Thailand (Waewsak, Landry, and Gagnon 2015)
FOWTs have been developed to overcome some or all of the limitations of onshore wind turbines ecosystem impacts, aesthetic impacts, and noise impacts (Jaber 2013). There are various benefits of offshore wind resources over onshore wind resources, including higher wind speeds, less turbulence, and higher stability, in terms of continuity of wind flow (Waewsak, Landry, and Gagnon 2015). By installing wind turbines in the ocean, the wind turbine capacity can be expanded to achieve greater energy collection. The power output can be increased by up to $40 \%$ compared with that achieved with onshore wind turbines. In addition, the noise and visual drawbacks of wind turbines can be eliminated by the installation of wind turbines in the ocean. Transportation simply involves towing floating platforms from their assembly points to selected offshore locations. Thus, FOWTs are potentially simpler and more economical to construct than onshore turbines, given prevailing transportation regulations (SNC - Lavalin 2018). Nevertheless, there are disadvantages to FOWTs, including their high costs and the difficulty and complexity of their maintenance. Helicopters or boats are required to carry the equipment and technicians to the sites. Furthermore, the necessary transmission lines are located onshore, which means that losses of electrical power in transmission cables can occur (Burger 2016). Hence, various applications have been created for utilizing FOWTs suitably, including water desalination plants, offshore power stations for ocean boats, and electrical power sources for islands and offshore drilling rigs (Roynarin 2016).

Thailand is located in an area of low wind speeds, with on-land average wind speeds of approximately $4-5 \mathrm{~m} / \mathrm{s}$ (Exell 1985). Fig. 1 presents the distribution of mean wind speeds at $40 \mathrm{~m}$ above sea level in the Gulf of Thailand. The mean offshore wind speeds in most areas of the Gulf are approximately $4-5.5 \mathrm{~m} / \mathrm{s}$, with a maximum mean wind speed of $6 \mathrm{~m} / \mathrm{s}$.

\subsection{Types of Wind Turbines}

Wind turbines can be classified according to the type of rotating axis of the rotor blades. There are two major types of modern wind turbines: horizontal-axis wind turbines (HAWTs) and vertical-axis wind turbines (VAWTs) (Rashid 2015). HAWTs have a rotating axis of the blade parallel to the wind flow. The system is operated using a tail vane or wind flow sensor to rotate the rotor blade to counter the wind direction. VAWTs rotate with respect to their vertical axis, which is perpendicular to the wind direction.
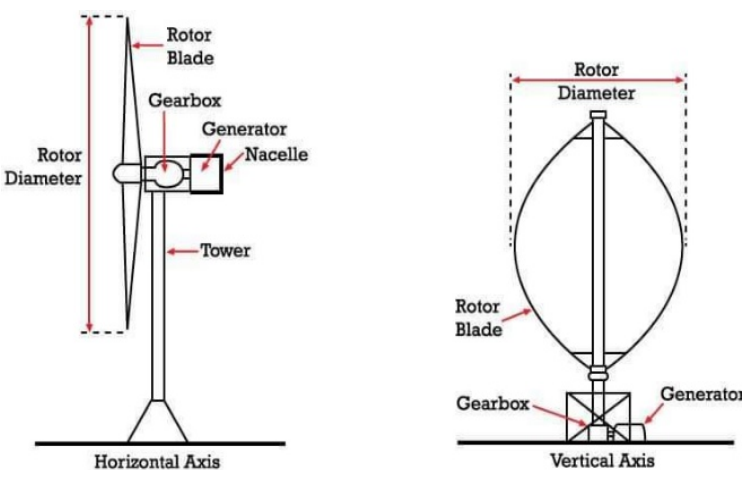

Fig. 2 Types of wind turbines (Mahmoud and Xia 2012) 
The turbine system can operate with any wind direction, with no tail vane or wind flow sensor required. In addition, VAWTs are simple to maintain because the generator and gearbox are located on the ground, as shown in Fig. 2. However, HAWTs are commercially more promising than VAWTs because of their greater power efficiency, low cutin wind speeds, and better power production economics (Clarke 2018; Tong 2010).

\subsection{Types of Floating Offshore Wind Turbines}

FOWTs can convert more wind power into electrical power than onshore turbines can, because of the greater wind resources available and continuity of wind flow in the ocean. Therefore, FOWTs can provide higher capacity factors than land-based wind turbines (Tong 2010). The essential factor that needs to be considered is the design of the floating platform, which should be strong enough to withstand the extreme weather conditions in oceans. Three types of floating platforms have been developed in prototype and commercial forms: the spar-buoy, sparsubmersible, and tension-leg platforms, as shown in Fig. 3 (International Renewable Energy Agency 2016).

A semi-submersible is a floating foundation constructed by connecting several columnar tubes that are supported by bracing members. Stability is supplied by the columnar tubes. The wind turbine tower is attached to either one of the columnar tubes or the center of the floating foundation (International Renewable Energy Agency 2016).

Spar is a floating foundation with a cylindrical ballast structure at the bottom of the foundation. Stability is achieved by the center of gravity being lower in the water than the center of buoyancy, since the upper parts of the foundation are lighter, and the lower parts are heavier. This type of structure is simple to fabricate and is highly stable. However, there are several difficulties associated with the assembly, transportation, and installation of such a structure, which requires very deep water for the large draft of the foundation (Costa Ros and James 2015).

A tension-leg platform (TLP) is a semi-floating foundation that uses tensioned mooring lines attached to the seabed to achieve adequate stability. The TLP design concept involves a structure that floats by virtue of buoyant force, with any attempt to push the structure above the water mitigated by the tensioned mooring lines that drag the structure down. Because of the low draft and tension stability of this type of structure, it can be lighter and smaller than other types. However, this type of structure could face risks during the operational phase because the design increases the stresses on the tendon and anchor system; failure can cause structural overturning (Taboada 2015).

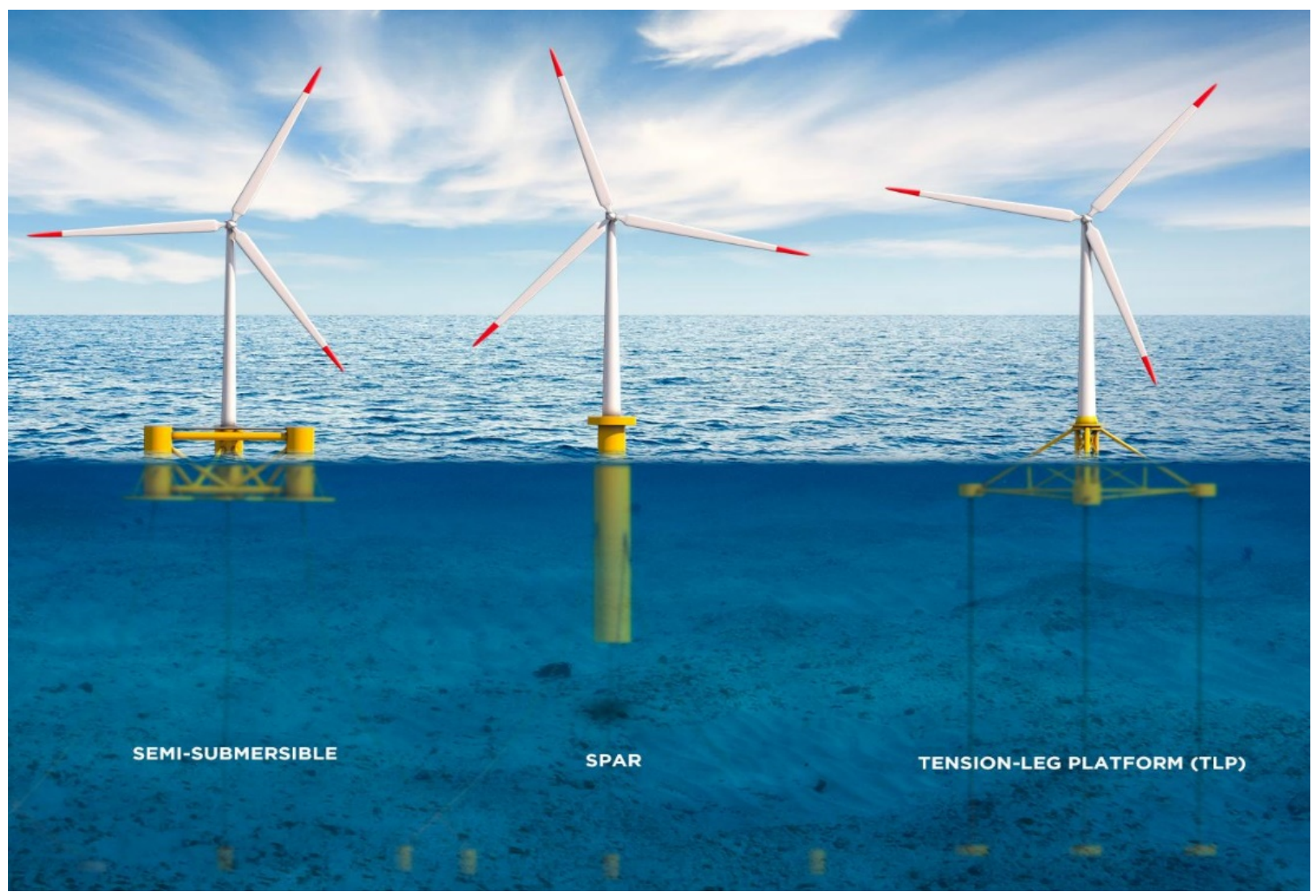

Fig. 3 Floating offshore wind turbine design concepts (Society for Underwater Technology 2018) 


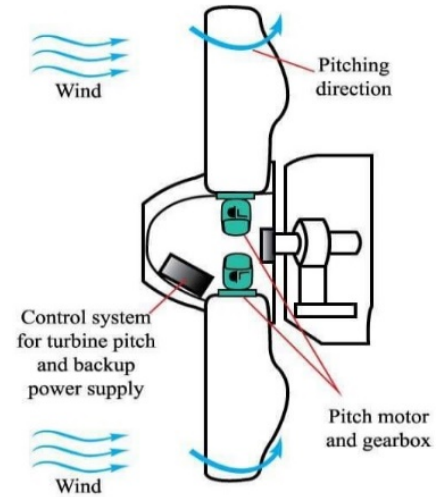

Fig. 4 Pitch control wind turbine (Jabir et al. 2017)

\subsection{Wind Turbine Controls}

A wind turbine control system is essential for ensuring that a wind turbine is operated effectively in terms of reliability, safety protection, and maximum power output. There are two main types of wind turbine control systems: pitch control systems and stall control systems (Tong 2010).

A pitch control system is an important mechanism in modern wind turbine technology. This type of system is operated by varying the blade pitch angle depending on the direction and intensity of the wind, as shown in Fig. 4. Therefore, a pitch control system optimizes wind turbine performance, in terms of wind energy conversion and power output stability, at any wind speed. In addition, this type of system can operate as an emergency system by regulating the wind turbine at high wind speeds to avoid any damage to the turbine (Rivkin, Anderson, and Silk 2013).

A stall control system is operated by stalling the blades after the wind turbines reach the rated wind speed. There are two types of stall control systems: passive control systems and active control systems. A passive control system is installed on a wind turbine by connecting the rotor blade to the hub at a fixed angle. Hence, the wind turbine performs at almost maximum efficiency at low wind speeds and is regulated by stalling the blades to control the rotational speed and power output to avoid the risk of damage and limit the rotational speed during high wind speeds. This control system is mainly designed for small-tomedium-sized wind turbines. In contrast, an active control system operates by stalling blades in conjunction with pitch control. At high wind speeds, the blades are turned toward a stall as the pitch control system operates in the opposite direction. This is called negative pitch control. This type of control system is mainly used for large wind turbines because of the ability of pitch control to maintain the rated power at high wind speeds and because of the accuracy of power output controls. However, the use of a pitch control mechanism adds to the cost of a wind turbine (Tong 2010).

\subsection{Wind Turbine Control Strategies}

The most effective control strategy for a wind turbine is to control the pitch angle and the generator. There are several factors that can affect the effectiveness of the control strategies of wind turbines, including the angle of attack and the power coefficient. In addition, the wind turbines optimization can be regulated via pitch, yaw, and rotational speed control. These control strategies are important in maximizing wind turbine performance and ensuring safe construction and operation of wind turbines (Rao 2019).
There are four types of control strategies for wind turbines: fixed-speed fixed-pitch (FS-FP) control, fixed-speed variable-pitch (FS-VP) control, variable-speed fixed-pitch (VS-FP) control, and variable-speed variable-pitch (VSVP) control. These control strategies are implemented to manage a wind turbine according to a power curve. Each control strategy provides a different power curve performance, as shown in Fig. 5 (NI 2019).

FS-FP control cannot improve performance via active control. Therefore, a high-speed passive stall method is used to regulate the wind turbine performance. The power line frequency and rotational speed are fixed by the generator speed because the generator is attached directly to the power grid. In addition, passive control is utilized for the gearbox ratio to avoid power overstepping. Fig. 5 shows that the energy capture is lower, which causes the actual power to be lower than the ideal power, as a result of the maximum efficiency being achieved at only one wind speed. Hence, the rated power applies to only one wind speed (Rao 2019).

FS-VP is a control strategy that achieves the maximum power efficiency at one wind speed. This strategy involves changing the pitch angle of the blades at wind speeds greater than the rated wind speed, whereas at wind speeds below the rated wind speed, the pitch angle is fixed. There are two FS-VP control methods that can be applied to constrain the power: feather control and stall control. The feather method increases the complexity of the control design, whereas stall control can increase unwanted thrust forces. Fig. 5 shows that below the rated wind speed, the power efficiency almost reaches the optimal efficiency in region 2. Hence, the pitch angle must be constantly adjusted to maintain power efficiency at speeds above the rated wind speed to prevent power loss (NI 2019).

VS-FP is a control strategy that uses power regulation to manage the speed of the generator, which is related to the wind speed. The airfoil blade design is an essential factor in fixed-pitch control of power via passive stalling. Fig. 5 shows that the optimal power efficiency is achieved at low wind speeds. Thus, there is only one wind speed that can produce the rated wind power. Passive stall control is important for regulating power as it avoids the rated power from being reached. This could be an unsuitable power control approach under some conditions. The VS-FP control strategy is suitable for extracting energy and achieving good power efficiency in areas with low wind speeds. VS-FP is well-known for its applicability in smallto medium-scale wind turbine designs as it is simple, economical, and highly reliable (Rao 2019; Jiawei, Changyun, and Yongduan 2014).

VS-VP is a combination of VS-FP and FS-VP. At wind speeds below the rated wind speed, VS-FP can be implemented to optimize power efficiency and improve power performance. In addition, FS-VP can be implemented at wind speeds above the rated wind speed to improve power control efficiency at the rated wind speed. This control strategy is the only approach that matches the ideal power curve (Rao 2019).

\subsection{Wind Turbine Parameters}

Tip speed ratio (TSR): TSR is a dimensional factor that reflects the relation between the blade tip speed and the actual wind speed. Its value can be determined by measuring the rotational speed and wind speed experimentally. 


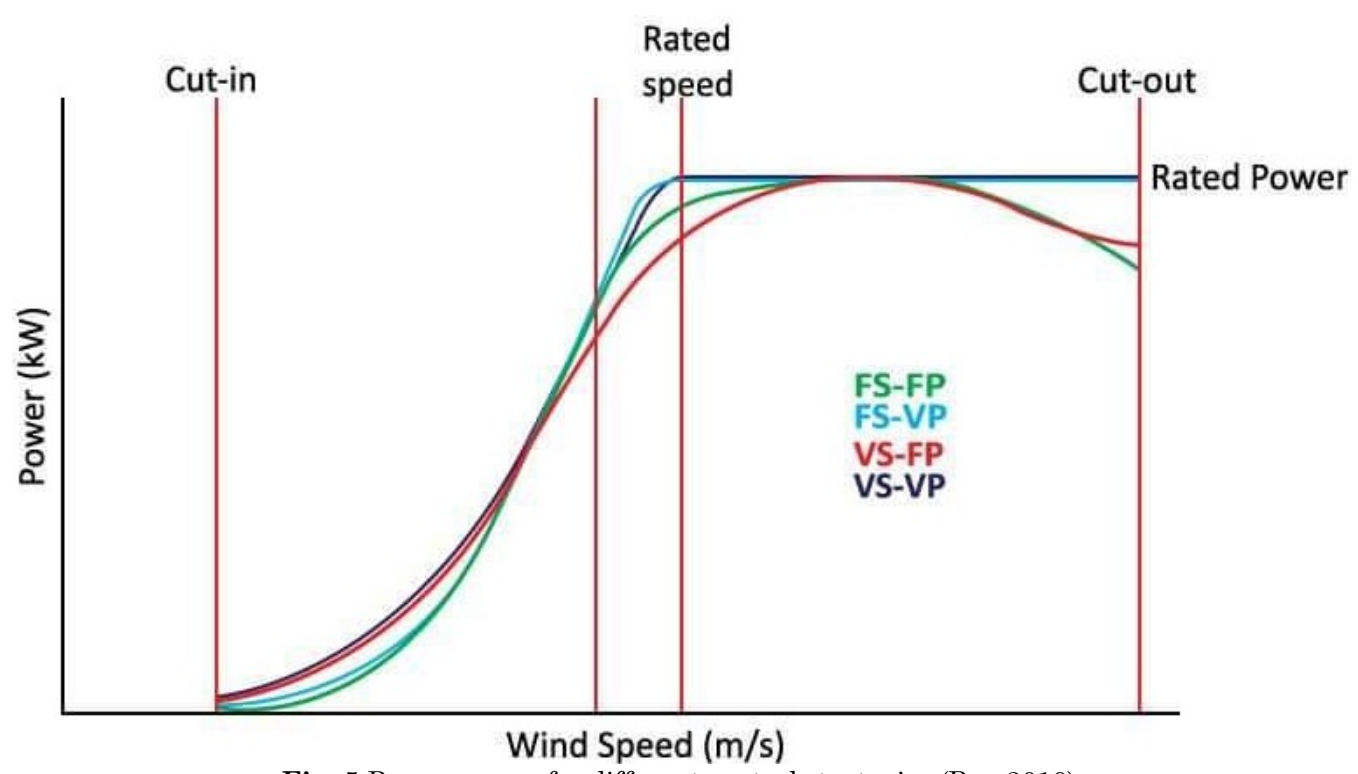

Fig. 5 Power curves for different control strategies (Rao 2019)

TSR can be used with the power coefficient $\left(\mathrm{CP}_{\mathrm{P}}\right)$-TSR curve in Fig. 9 to determine the $\mathrm{CP}_{\mathrm{P}}$ value of the wind turbine at a specific wind speed. The equation for TSR can be written as follows (Cao 2011):

$$
T S R=\frac{U}{V}=\frac{\omega r}{V}=\frac{2 \pi N r}{V 60}
$$

where $\mathrm{U}$ is the velocity of the rotor tip $(\mathrm{m} / \mathrm{s}) ; \mathrm{V}$ is the wind speed $(\mathrm{m} / \mathrm{s}) ; \omega$ is the angular velocity $(\mathrm{rad} / \mathrm{s}) ; \mathrm{r}$ is the rotor radius $(\mathrm{m})$; and $\mathrm{N}$ is the rotational speed of the rotor (rpm).

Wind power output: A wind turbine converts kinetic energy from wind into electrical power. The primary factors that determine the wind turbine power output are the rotor blade size and wind speed. The power output equation can be written as follows (Manwell, J. F., McGowan, J. G., Rogers 2009):

$$
P_{w}=\frac{1}{2} \rho A V^{3} C_{P}
$$

where $\rho$ is the air density $\left(1.225 \mathrm{~kg} / \mathrm{m}^{3}\right)$; A is the swept area $\left(\mathrm{m}^{2}\right) ; \mathrm{V}$ is the wind speed $(\mathrm{m} / \mathrm{s})$; and $\mathrm{C}_{P}$ is the power coefficient of the wind turbine.

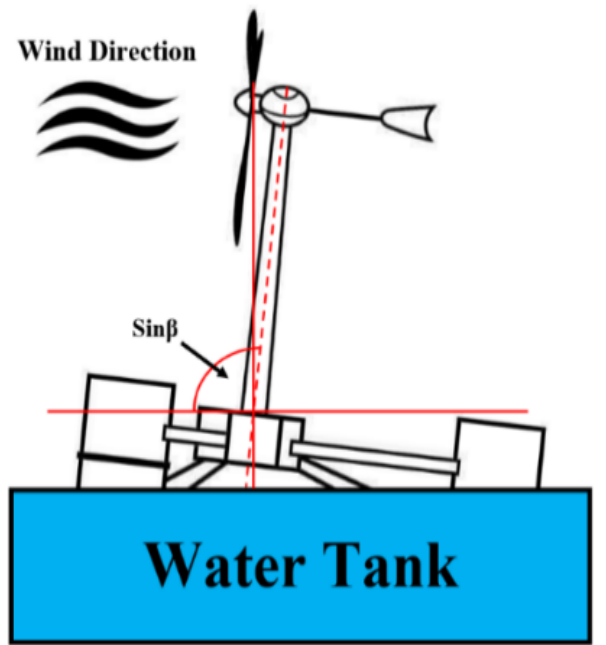

Fig. 6 Tilt angle in FOWT power output equation
FOWT performance can be adversely affected by the tilt angle, which results from vertical misalignment of the wind turbine origin position with respect to the wind flow. This can affect the effective area available to capture energy from the wind flow. Fig. 6 shows that the reduction factor is the greatest at $\sin \left(90^{\circ}\right)=1$, when the wind turbine blade is positioned at a stationary point and is perpendicular to the wind direction. The reduction factor decreases as the tilt angle increases, which degrades the wind turbine performance. Hence, the reduction factor can be determined as $\sin \left(90^{\circ}+\right.$ tilt angle), which is assumed to be $\operatorname{Sin} 6$ in the FOWT power output equation:

$$
P_{\text {FOWT }}=\frac{1}{2} \rho A V^{3} C_{P} \operatorname{Sin} \beta
$$

\subsection{Computational Fluid Dynamics}

Computational fluid dynamics (CFD) is a computer simulation system for analyzing fluid engineering systems using mathematical models. CFD can be used to simulate wind turbine performance and analyze wind flow behavior over wind turbines to determine how to obtain optimal results. CFD results can be compared with experimental results to ensure precise outcomes and identify errors that can occur during experiments (Autodesk Knowledge Network 2019).

The CFD modeling process consists of three stages: preprocessing, solution, and post-processing. Pre-processing involves assigning the governing equations, properties, and boundary conditions within a domain composed of small interconnected volumetric elements to create a fluid motion model. Temporal and spatial discretization patterns are chosen for the governing equations in the solution stage. In addition, there are several factors that must be determined, such as the convergence criteria or number of iterations allowed, relaxation factors, and the pressure and velocity coupling algorithm. Post-processing involves analyzing and interpreting the results for various flow, temperature, and pressure conditions (Alonzo-García, Gutiérrez-Torres, and Jiménez-Bernal 2016). 


\subsection{Turbulence Model}

Selection of an appropriate physical model can be considerably difficult in the case of turbulent flow. The equations of an ideal turbulence model should involve the minimum number of complexities possible while adequately reflecting the physical factors related to the phenomenon being modeled (Sodja 2007).

Turbulence modeling is a process for closing the mean flow equations system. Turbulent fluctuation modeling is not essential for correcting the solution results for all problem cases, but the effects that turbulence has on the mean flow should be determined. Turbulence models are widely used in various applications involving precise data in a simple and cost-effective manner. Commonly used turbulence models are based on Reynolds-averaged (i.e., time-averaged) Navier-Stokes (RANS) equations. For instance, a zero-equation system would use a mixing length model; a one-equation system would employ the SpalartAlmaras model; and a two-equation system would use $\mathrm{k}-\varepsilon$ style models (standard, re-normalization group (RNG), or realizable) and a $\mathrm{k}-\omega$ model (Bakker 2002).

The $\mathrm{k}$-epsilon $(\mathrm{k}-\varepsilon)$ model is the most widely used model for describing the mean flow features in a turbulence model. This type of model is used in a two-equation system as an adjunct to the Navier-Stokes equations. There are two variables in this type of model that describe the ordinary features of turbulence characteristics in a two-equation system: the turbulence kinetic energy (k) and the dissipation rate of turbulence kinetic energy $(\varepsilon)$. This type of model is applicable to free-shear flows with relatively small pressure gradients. In addition, this type of model is quite simple to use as it only requires inputs for the initial values and/or boundary conditions (Argyropoulos and Markatos 2015; Simscale 2020).

The $\mathrm{k}-\varepsilon$ model equations can be written as follows (Poradowski 2013):

$\mathrm{k}$-transport equation

$\rho \frac{D k}{D t}=\frac{\partial}{\partial x_{j}}\left[\left(\mu+\frac{\mu_{t}}{\sigma_{k}}\right) \frac{\partial_{k}}{\partial x_{j}}\right]+\mu_{t} S^{2}-\rho \varepsilon ; S=\sqrt{2 S_{i j} S_{i j}}$

$\varepsilon$-transport equation

$\rho \frac{D \varepsilon}{D t}=\frac{\partial}{\partial x_{j}}\left[\left(\mu+\frac{\mu_{t}}{\sigma_{\varepsilon}}\right) \frac{\partial_{\varepsilon}}{\partial x_{j}}\right]+\frac{\varepsilon}{k}\left(C_{1 \varepsilon} \mu_{t} S^{2}-\rho C_{2 \varepsilon} \varepsilon\right)$

Coefficient

$\sigma_{k}, \sigma_{\varepsilon}, C_{i \varepsilon}, C_{\varepsilon 2}$

Note: Air and water are used in simple flow experiments to determine the values of these constants.

Turbulent viscosity

$$
\mu_{t}=\rho C_{\mu} \frac{k^{2}}{\varepsilon}
$$

\subsection{Governing Equations}

CFD is based on the governing equations of fluid dynamics, which are derived from the mathematical equations of continuity, the conservation laws of physics, and the Navier-Stokes equations. There are three main physical laws underlying the governing equations: the continuity equation (the conservation of mass), the conservation of momentum according to Newton's second law, and the conservation of energy according to the first law of thermodynamics (Tey et al. 2017; Tu, Yeoh, and Liu 2018). The general forms of the governing equations are as follows (Munson et al. 2009):

\section{Continuity equation}

$\frac{\partial \rho}{\partial t}+\nabla \cdot(\rho \vec{V})=0$

Conservation of momentum

$\rho \frac{\partial \vec{V}}{\partial t}+\rho(\vec{V} \cdot \nabla) \vec{V}=-\nabla \rho+\rho \vec{g}+\nabla \cdot \tau_{i j}$

Conservation of energy

$\frac{\partial}{\partial t} \int e * \rho d \forall+\int\left(\breve{u}+\frac{p}{\rho}+\frac{V^{2}}{2}+g z\right) \rho V \cdot \breve{n} \mathrm{dA}=Q_{\text {net in }}^{\cdot}+W_{\text {net in }}^{\cdot}$ (9)

The solutions can be calculated using partial differentials, which is the numerical method employed in CFD. There are several techniques for simplifying the problems by making some assumptions, such as is steady, isothermal, and incompressible fluid flow. In addition, some problems can be simplified by eliminating certain variables. A finite volume is used in CFD for simulation and spatial discretization of the governing equations. The numerical method must be verified.

\section{Experimental}

\subsection{Experimental Model Description}

Experiments were conducted in a wind tunnel at the Rajamangala University of Technology Thanyaburi (RMUTT) Energy Research and Service Centre. The wind tunnel was $3 \mathrm{~m}$ high, $4 \mathrm{~m}$ wide, and $4.5 \mathrm{~m}$ in length, with a $1 \mathrm{~m} \times 1 \mathrm{~m}$ airflow duct in the middle. The wind tunnel contained a 20,000-CFM centrifugal fan with a fan speed controller and a water tank for use with a FOWT model, as shown in Fig. 8.

In this study, the performance of a VS-FP FOWT was analyzed by comparing the results obtained via wind tunnel testing of an experimental model with CFD simulation model results. The experimental model was tested at wind speeds of $2.0-5.5 \mathrm{~m} / \mathrm{s}$. A semi-submersible floating platform model was used because of its stability and simplicity of construction and because of the limited available space in the wind tunnel.

There are two possible FOWT platform positions in the water tank: two columnar tubes at the front, facing the wind flow, and two columnar tubes at the back of the platform, as shown in Fig. 9. The platform position with two columnar tubes at the front was used in this experiment because the platform position with two columnar tubes at the back may potentially provide a higher buoyant force, which means that it would provide more support at the column tubes to reduce the effect of the tilt angle. The platform position with two columns at the front is affected more by the tilt angle because the columnar tubes at the back provide less buoyant force. 


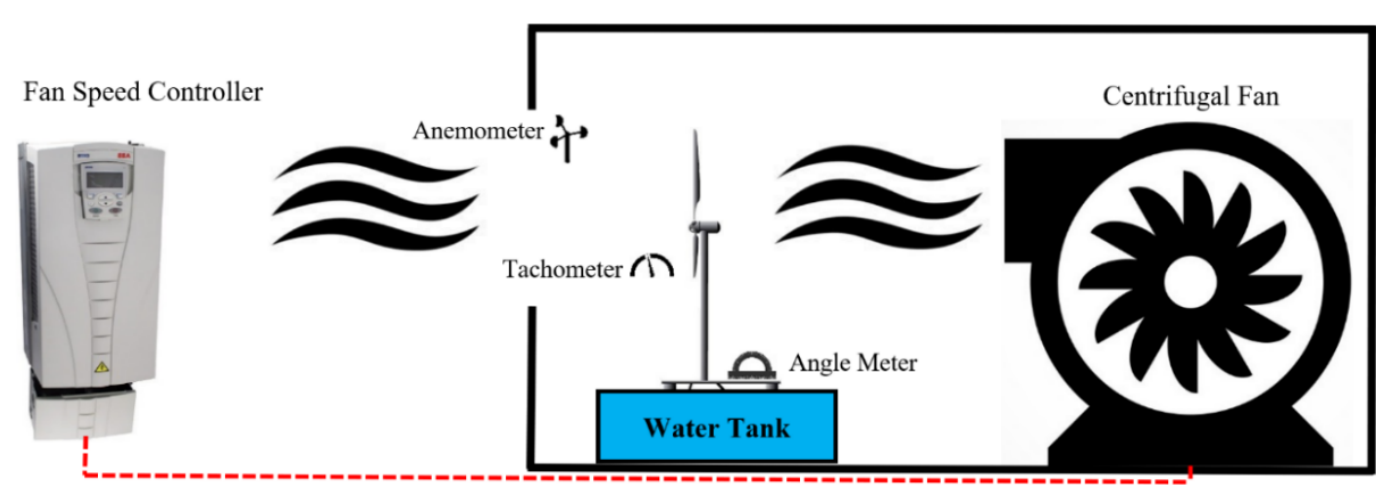

Fig. 8 Schematic diagram of wind tunnel
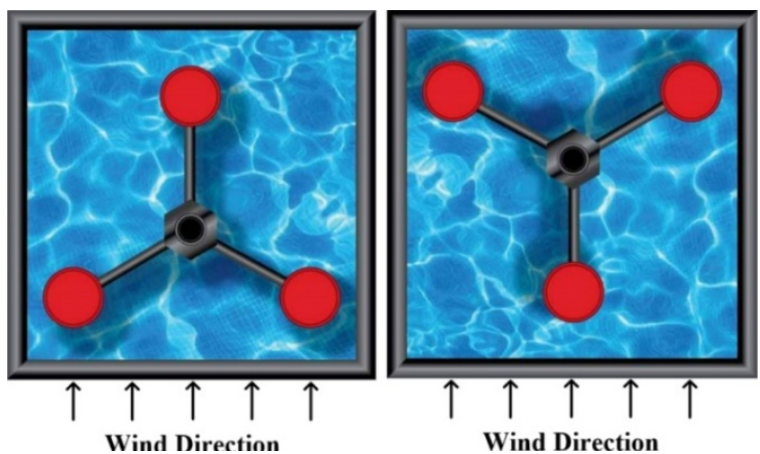

Fig. 9 FOWT platform positions: Left: Two columnar tubes at the front. Right: Two columnar tubes at the back

Thus, this experiment investigated the critical effect of the tilt angle in analyses of the worst-case scenario for FOWTs. Experimental data were obtained using three measurement tools: an anemometer for measuring the wind speed, a tachometer for measuring the rotational speed, and an angle meter for measuring the tilt angle. The wind flow in the wind tunnel was created by the centrifugal fan pulling air through the duct. The fan speed controller was used to adjust the wind speed.

The R1235 airfoil blade profile was used in the experiment model. This blade profile, which was designed to be used in areas with low wind speeds, creates a high lift force at low Reynolds-number flows. The cut-in speed of the R1235 blade profile is $2 \mathrm{~m} / \mathrm{s}$, with an average wind speed of 4-5 m/s. It produces a high TSR and a maximum power coefficient at 0.35 , as seen in Fig. 10 .

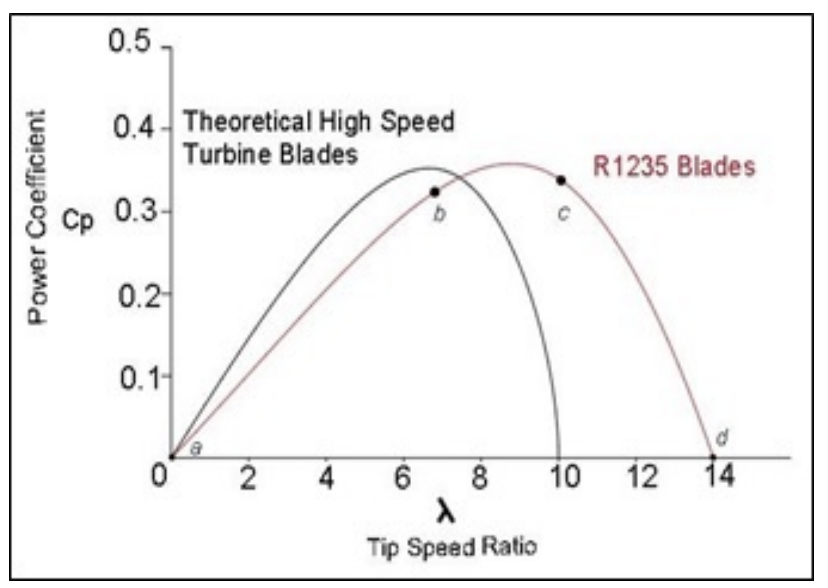

Fig. 10 R1235 airfoil blade tip speed ratio (Roynarin et al. 2020)

\subsection{CFD Boundary Conditions}

The CFD simulation program CFDesign v7 was used in this study. The experimental test results obtained were compared with those obtained via CFD modeling. Fig. 11 shows the boundary conditions applied to the CFD model for a wind tunnel $5 \mathrm{~m}$ wide and $10 \mathrm{~m}$ long and a rotating region $1 \mathrm{~m}$ wide. A set distance is required before a wind turbine can have a marked impact on a rotor blade; therefore, the rotor blade was placed at a distance of $7 \mathrm{~m}$ from the velocity inlet to allow laminar wind flow. The standard $k-\varepsilon$ turbulence model was applied in the simulation. Two types of boundary conditions were applied. The first type included fixed conditions for the objects in the three-dimensional model that are stationary when a force is applied, i.e., the wind tunnel wall and edges. The second type constituted the moving conditions, which are applied to objects that move when acted upon by forces. In the experiments conducted in this study, the wind turbine blades (the rotating region) and moving air were subjected to moving boundary conditions. The rotating region is a part of a motion module used for analyzing a rotating device and encompasses the spinning object. Table 1 shows the CFD model parameters and values applied to the control volume model to solve the problem. The standard $\mathrm{k}-\varepsilon$ model was used as the turbulence model.

\subsection{Mesh Refinement}

The Eulerian specification of the flow field was adopted in the CFD model to analyze moving fluid at a particular location in space through which fluid flows over a fixed mesh. 
The cell-vertex numeric was applied to separate the domain, which could result in unstructured tetrahedral elements. Therefore, the complicated rotating geometry was to be detected; automatic meshing was used for further geometry improvements. There are three steps for mesh adaptation to adjust for high-volume velocity gradients in problem analyses: coarse meshing, medium meshing, and fine meshing, as shown in Fig. 12 (Kongkapisuth, Roynarin, and Intholo 2017).

Fig. 13 visually shows the suitable amounts of meshing for the CFD wind turbine model for various blade velocities, determined by testing the wind speed model at an inlet wind speed of $5.5 \mathrm{~m} / \mathrm{s}$. The graph shows the relationship between the number of elements and the rotational speed of the wind turbine over a range of 12,000 to $2,500,000$ elements. As can be observed, the slope becomes constant when the number of model elements reaches 1,500,000. Therefore, 1,500,000 elements were used in the CFD simulation model. Fig. 14 shows that the meshing of each part is not the same as the element size. The blade element size should be the most detailed due to the complexity of the curvature of the blade surface. The complex airfoil surface needs fine meshing to make the elements smooth along the surface of the airfoil.

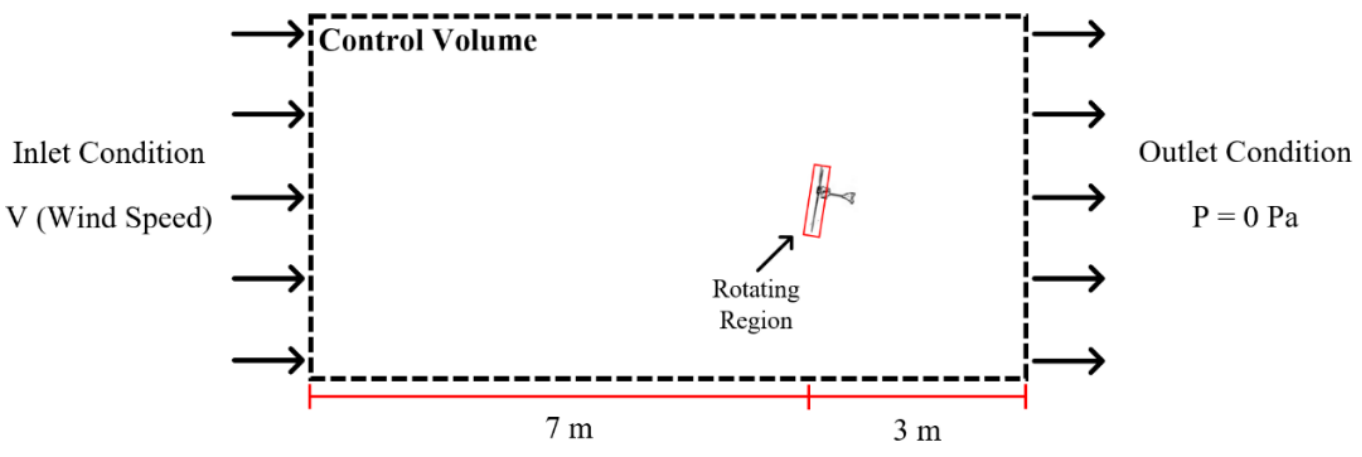

Fig. 11 CFD boundary conditions

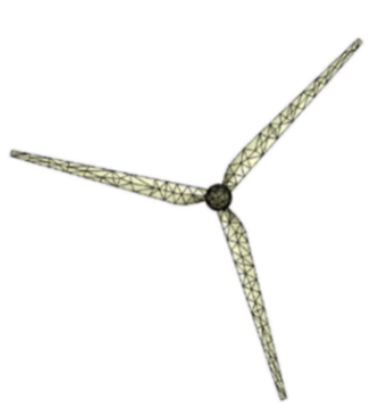

(a) Coarse mesh

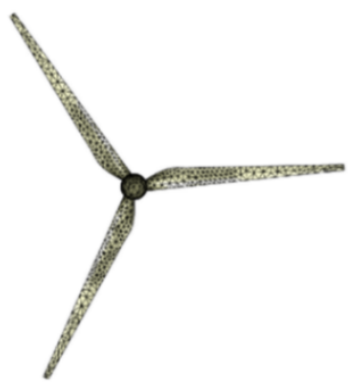

(b) Medium mesh

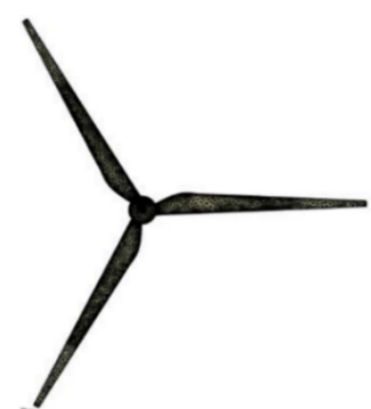

(c) Fine mesh

Fig. 12 Wind turbine blade meshing in CFD

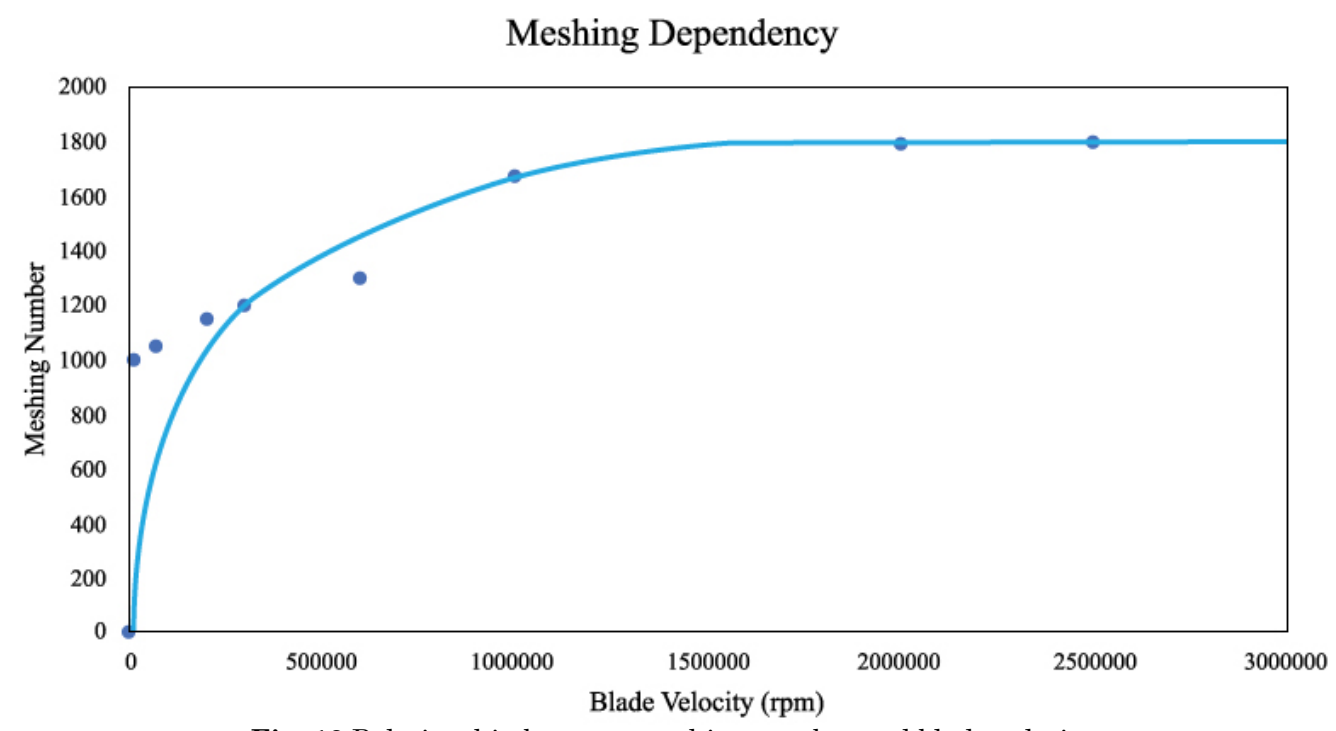

Fig. 13 Relationship between meshing number and blade velocity 


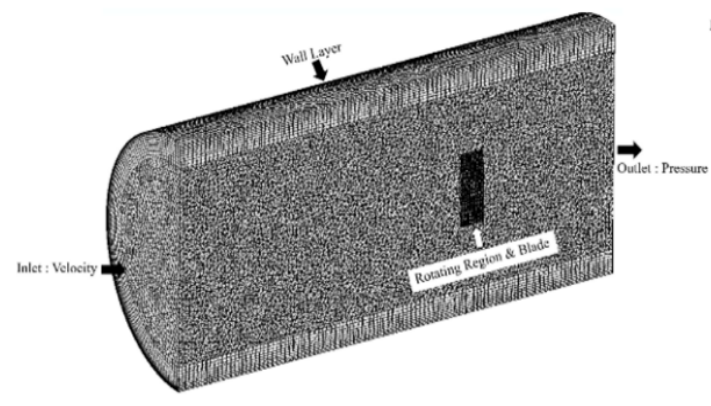

(a)

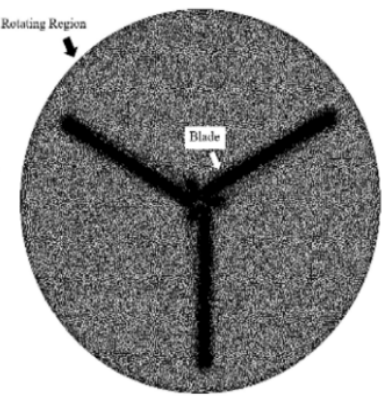

(b)

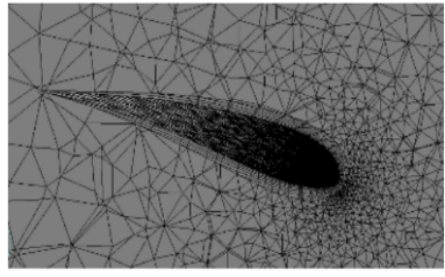

(c)

Fig. 14 CFD meshing structure: (a) Meshing of cross-section of wind tunnel; (b) Meshing of blade rotation region; (c) Meshing of airfoil cross-section

Table 1

CFD model parameters

\begin{tabular}{ccc}
\hline Parameter & Value & Units \\
\hline Inlet: Wind Speed & $2-5.5$ & $\mathrm{~m} / \mathrm{s}$ \\
Inlet Total Temperature & 320 & $\mathrm{~K}$ \\
Angular Velocity & Free Spin & $\mathrm{rad} / \mathrm{s}$ \\
Working Fluid & Ideal Air & $\mathrm{kg} / \mathrm{m}^{3}$ \\
Fluid Density & 1.225 & \\
Turbulence Model & $\mathrm{k}-\varepsilon$ & $\mathrm{Pa}$ \\
Outlet Pressure & 0 & \\
\hline
\end{tabular}

\section{Results and Discussion}

The wind turbine R1235 airfoil blade with $80 \mathrm{~cm}$ diameter was tested in the wind tunnel with a flow inlet of $1 \mathrm{x} 1 \mathrm{~m}$. The blockage ratio was measured as the ratio of the wind turbine swept area to the wind tunnel cross-sectional area (Eltayesh et al. 2019). Although the wind tunnel had a $50 \%$ blockage ratio, it had a negligible effect on the experimental results due to the $16.4 \%$ error with CFD.

The CFD velocity magnitude contour results obtained from using rotor tilt angles of $3.5^{\circ}-6.1^{\circ}$ and wind speeds between $2-5.5 \mathrm{~m} / \mathrm{s}$ are shown in Fig. 15. The figure displays the type of wind flow behavior, which had an impact on the rotor blade during CFD. The wind speed was reduced after impact with the rotor blade, causing a weak stream of air flow that was increased at higher inlet wind speeds. This resulted in observable weak flow regions and clearly separated turbulent flow layers.

The static pressures from the CFD are shown in Fig 16, illustrating that static pressure occurred when the wind flow made impact with the rotor blade. At the front of the rotor blade, higher pressures were found to be dependent on higher wind flows via increase in inlet wind speed. The back of the rotor blade was observed to produce negative pressure.

Fig. 17 shows a graph of the rotational speeds for the experimental model and CFD model for wind speeds of 2$5.5 \mathrm{~m} / \mathrm{s}$. A linear trendline analysis of the data shown in this graph was conducted. The graph shows that the CFD simulation results involved higher rotational speeds than the experimental results did, with average percentage differences of $16.4 \%$. This is because the CFD simulation only considers the rotation region of the wind turbine, which is the rotation of the rotor blade. In contrast, the rotor blade in the experiment is connected to the shaft axle and bearings to ensure that it can rotate when the wind blows. Hence, friction at the shaft axle and bearings could reduce the rotational speed of the experimental rotor blade.

Fig. 18 graphically depicts the TSR versus power coefficient plots for the experimental and CFD models. The possible power coefficient value was estimated using the tip speed ratio and the $\mathrm{R} 1235$ airfoil blade tip speed ratio graph in Fig. 10. The results are similar for power coefficient values in the range of $0.32-0.36$. However, the power coefficient values for the experimental and CFD models started to decrease after a TSR of 10 , which could have resulted from the rotor blades rotating quickly and thus gradually reducing the efficiency of wind power extraction, as the rotor blade would increasingly act as a solid wall preventing wind flow when the blade spins quickly. The shaded area in Fig. 15 shows that optimal power coefficient values of $0.35-0.36$ are obtained at TSRs of 7.7-9.6. In addition, the optimal power coefficient range corresponds to tilt angles of $3.9-5.8^{\circ}$ with wind speeds of $3-5 \mathrm{~m} / \mathrm{s}$, which are the average wind speed values in Thailand. In addition, the tilt angle would behave as variable pitch to maintain the optimal power coefficient, which could reduce the cost of pitch control systems for small to medium fixed pitch FOWTs. 
Citation: Wisatesajja, W., Roynarin, W., Intholo, D. (2021) Analysis of Influence of Tilt Angle on Variable-Speed Fixed-Pitch Floating Offshore Wind Turbines for Optimizing Power Coefficient Using Experimental and CFD Models. Int. Journal of Renewable Energy Development, 10(2), 201-212, doi: 10.14710/ijred.2021.33195

P a g e $\mid 210$

Velocity Magnitude $(\mathrm{m} / \mathrm{s})$

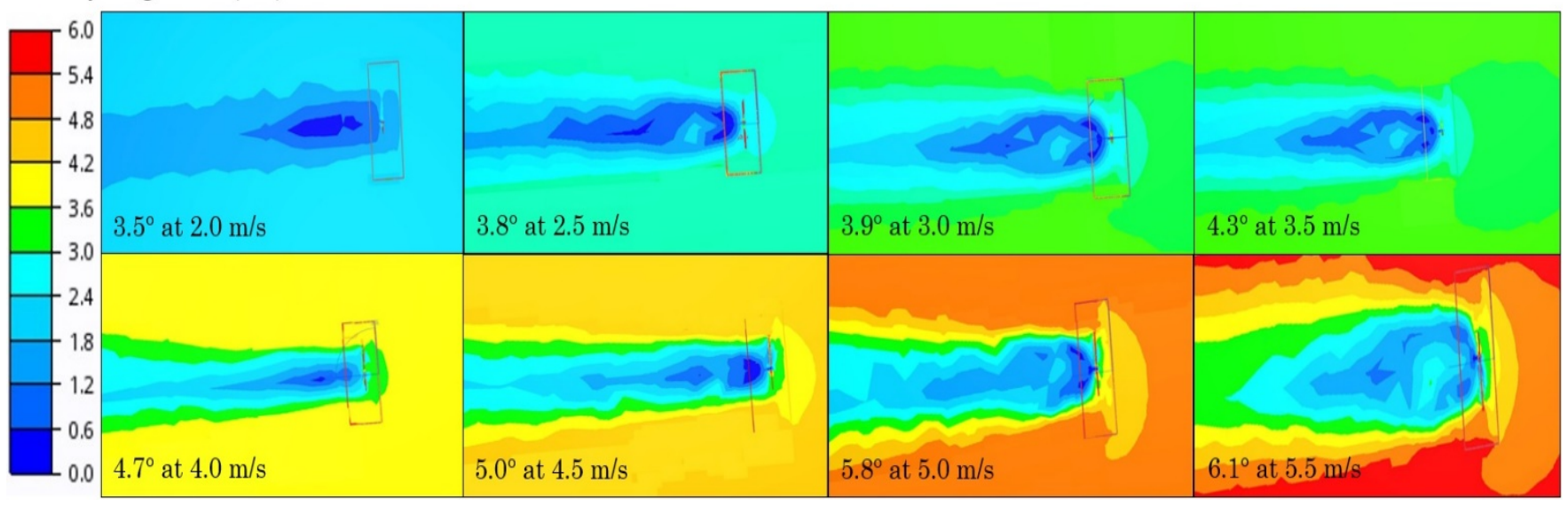

Fig. 15 Velocity magnitude

Static Pressure $(\mathrm{Pa})$

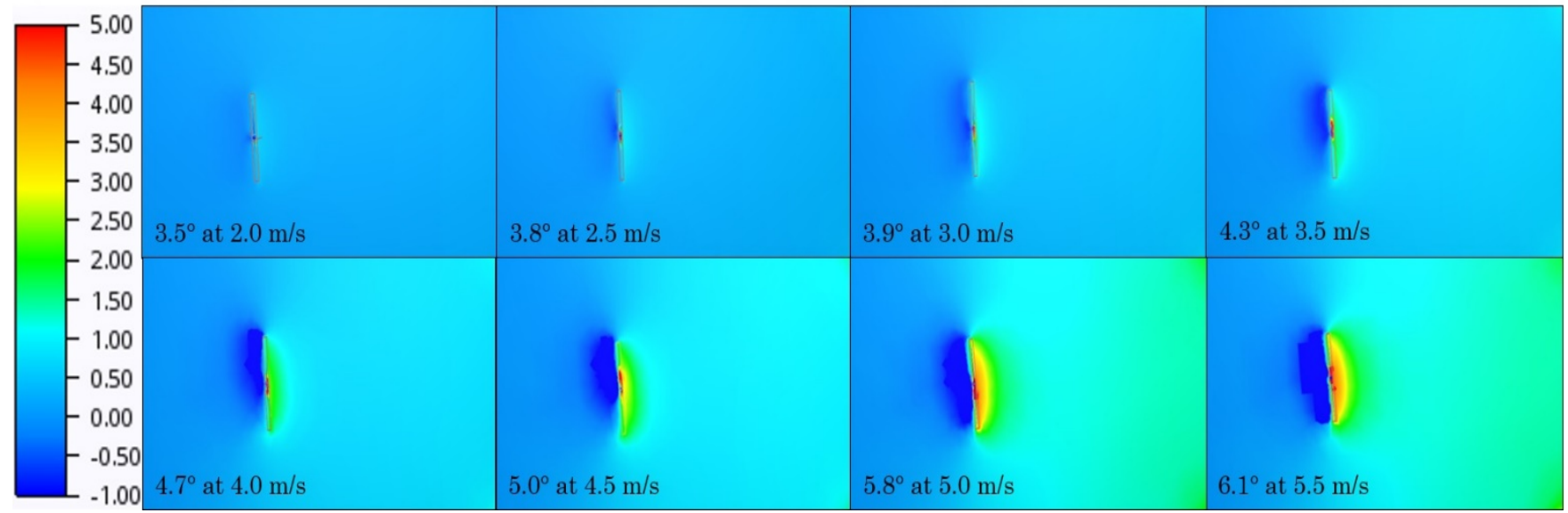

Fig. 16 Static pressure

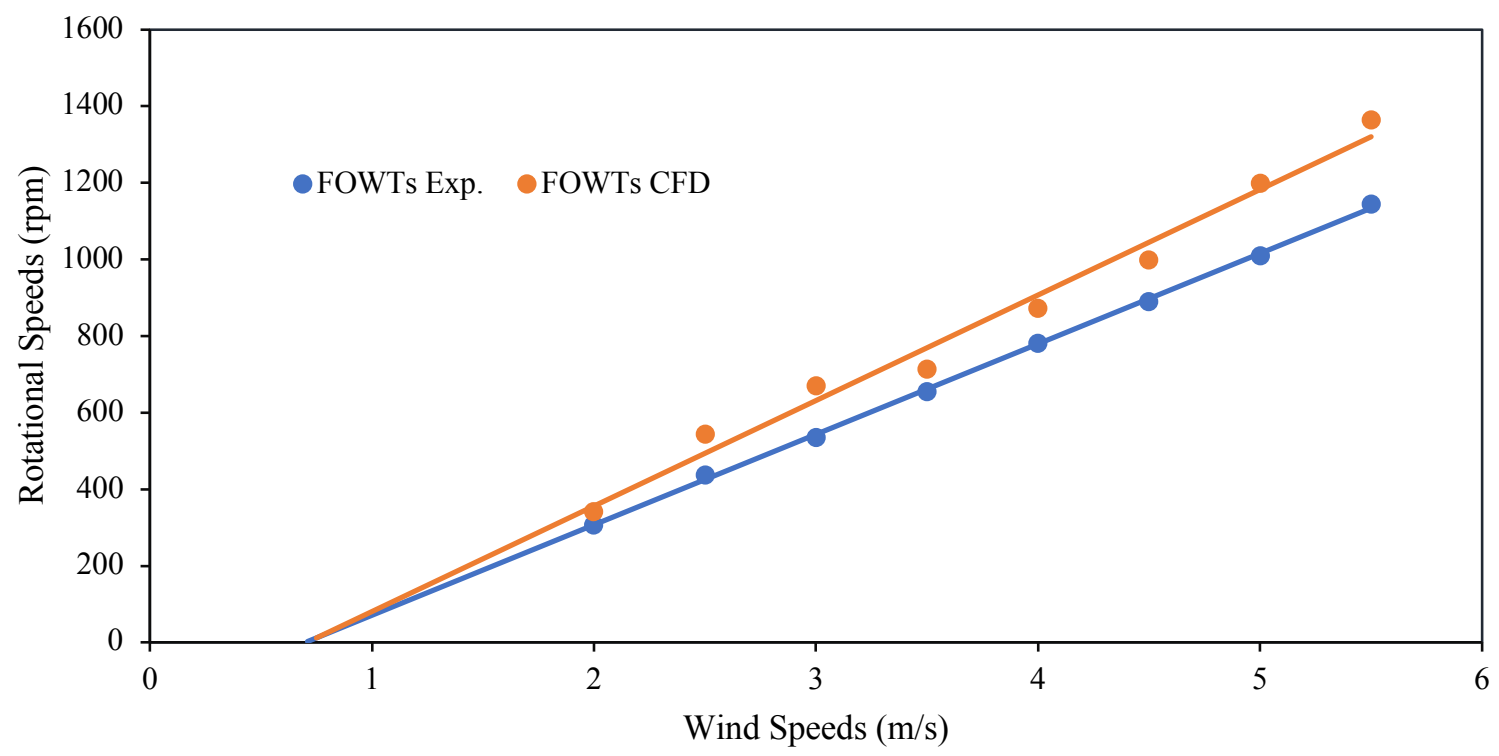

Fig. 17 Wind speed vs. rotational speed 


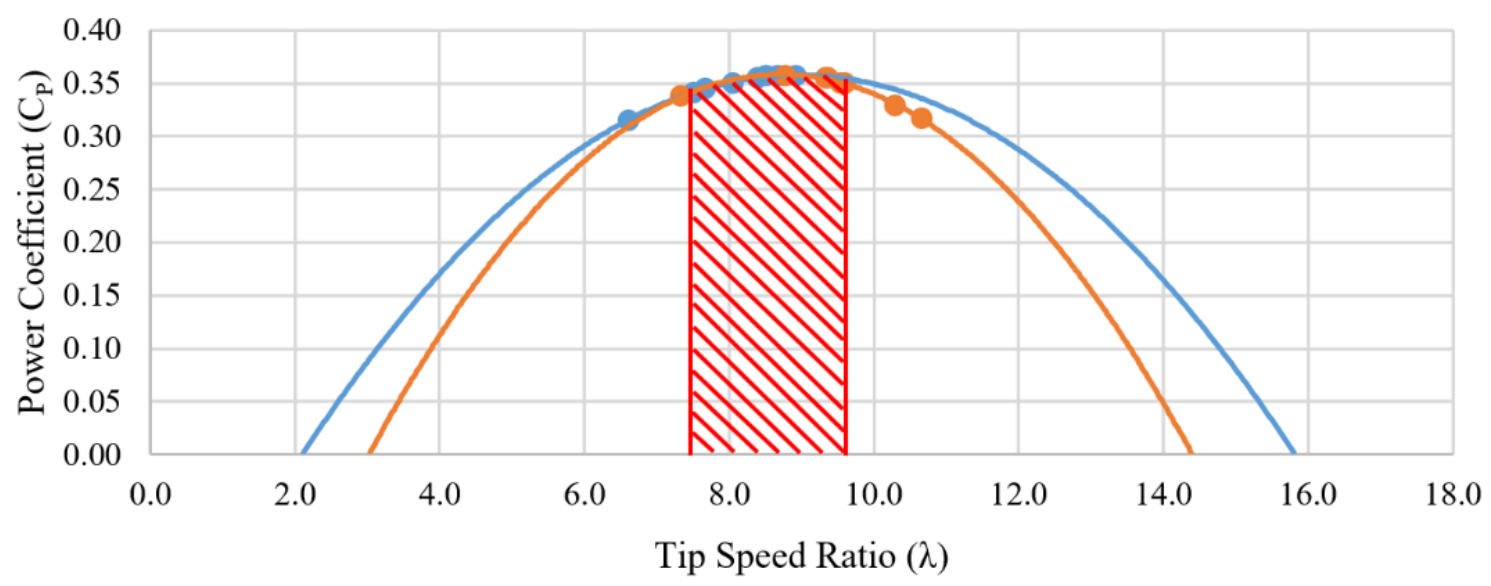

-FOWTs Exp. $\quad$ FOWTs CFD

Fig. 18 Wind speed vs. power coefficient

\section{Conclusions}

This study investigated the optimization of the power coefficient to maintain the wind power performance of FOWTs with variable-speed fixed-pitch control. An experimental model tested in a wind tunnel and a CFD simulation model were used for analysis and comparison, with wind speeds of $2-5.5 \mathrm{~m} / \mathrm{s}$ and tilt angles of $3.5-6.1^{\circ}$. The velocity magnitude contour and static pressure from CFD results are presented. The results show that average rotational speed differences of $16.4 \%$ and optimal power coefficients of $0.35-0.36$ can be maintained at tip speed ratios of 7.7-9.6 for wind speeds of $3-5 \mathrm{~m} / \mathrm{s}$ with tilt angles of $3.9-5.8^{\circ}$. The results of this study provide insights into a new concept of power coefficient optimization using variable tilt angle for small to medium fixed pitch FOWTs. The application of such wind turbines may potentially reduce the cost of pitch control systems.

In future research, this optimum power coefficient technique could be applied to R1235 wind turbine commercial products with generator torque loads to investigate the effects of the tilt angle on different aspects of FOWT performance, including the rotational speed, tip speed ratio, and power output. This would facilitate comparisons with on-land wind turbines in terms of economic value and performance.

\section{Acknowledgments}

I would like to thank my supervisor, Asst. Prof. Dr. Wirachai Roynarin, for the valuable knowledge and advice provided throughout this research. I would also like to thank my colleague, Mr. Decha Intholo, for his support and consultation on this paper. I am grateful to the staff of the Energy Research and Service Centre (ERSC) of the Faculty of Engineering of RMUTT for allowing me to use the experiment resources. Finally, I would like to thank my family for their consistent support.

\section{References}

Alonzo-García, A., Gutiérrez-Torres, C.C., \& Jiménez-Bernal, J.A. (2016). Computational Fluid Dynamics in Turbulent Flow
Applications. Numerical Simulation, p. 1839. DOI: $10.5772 / 63831$

Argyropoulos, C.D., \& Markatos, N.C. (2015). Recent Advances on the Numerical Modelling of Turbulent Flows. Applied Mathematical Modelling, 39(2), 693-732; DOI: 10.1016/j.apm.2014.07.001

Autodesk Knowledge Network. (2019). Fluid Flow Definitions. https://knowledge.autodesk.com/support/cfd/learn-

explore/caas/CloudHelp/cloudhelp/2014/ENU/SimCFD/files/G UID-4DAF0D6D-F1F4-4E90-A9C8-5CACB85E79BEhtm.html. Accessed on 26 April 2020.

Bakker, A. (2002). Lecture 10 - Turbulence Models: Applied Computational Fluid Dynamics. http://www.bakker.org/dartmouth06/engs150/10-rans.pdf. Accessed on 26 April 2020.

Burger, T. (2016) Pros and Cons of Floating Platforms in Marine Renewable Energy. https://pmiind.com/pros-cons-floatingplatforms-marine-renewable-energy/. Accessed on 14 April 2020.

Cao, H. (2011). Aerodynamics Analysis of Small Horizontal Axis Wind Turbine Blades by Using $2 D$ and $3 D$ CFD Modelling. MSc thesis, School of Computing, Engineering and Physical Science, University of Central Lancashire, Preston, United Kingdom.

Clarke, S. (2018). Electricity Generation Using Small Wind Turbines for Home or Farm Use. http://www.omafra.gov.on.ca/english/engineer/facts/18005.pdf. Accessed on 15 April 2020.

Costa Ros, M., \& James, R. (2015). Floating Offshore Wind: Market and Technology Review. https://prod-drupalfiles.storage.googleapis.com/documents/resource/public/Floati ng\%20Offshore\%20Wind\%20Market\%20Technology\%20Revie w\%20-\%20REPORT.pdf. Accessed on 22 April 2020.

Eltayesh, A., Hanna, M.B., Castellani, F., Huzayyin, A.S., ElBatsh, H.M., Burlando, M., \& Becchetti, M. (2019) Effect of Wind Tunnel Blockage on the Performance of a Horizontal Axis Wind Turbine with Different Blade Number. Energies, 12(10), 1988; DOI: 10.3390/en12101988

Exell, R. H. B. (1985). The Wind Energy Potential of Thailand. Solar Energy, 35(1), 3-13; DOI: https://doi.org/10.1016/0038092X(85)90031-3

Gumula, S., Piaskowska-Silarska, M., Pytel, K., Noga, H., \& Kulinowski, W. (2017). Evaluation of the Impact of Adjusting the Angle of the Axis of a Wind Turbine Rotor Relative to the Flow of Air Stream on Operating Parameters of a Wind Turbine Model. E3S Web of Conferences, 14, 10-20; DOI: https://doi.org/10.1051/e3sconf/20171401016

International Renewable Energy Agency (IRENA). (2016). Floating Foundations: A Game Changer for Offshore Wind Power. https://www.irena.org/- 
/media/Files/IRENA/Agency/Publication/2016/IRENA_Offshor e_Wind_Floating_Foundations_2016.pdf. Accessed on 17 April 2020 .

International Renewable Energy Agency. (2019). Renewable Energy Statistics 2019. https://www.irena.org//media/Files/IRENA/Agency/Publication/2019/Jul/IRENA_Ren ewable_energy_statistics_2019.pdf. Accessed 12 April 2020.

Jaber, S. (2013). Environmental Impacts of Wind Energy. Journal of Clean Energy Technologies, 1(3), 251-254; DOI: 10.7763/JOCET.2013.V1.57

Jabir, M., Illias, H.A., Raza, S., \& Mokhlis, H. (2017). Intermittent Smoothing Approaches for Wind Power Output: A Review. Energies, $\quad 10(10), \quad 1572 ; \quad$ DOI: https://doi.org/10.3390/en10101572

Jiawei, C., Changyun, W., \& Yongduan, S. (2014). Power Control Strategy for Variable-Speed Fixed-Pitch Wind Turbines. Proceedings of 13th International Conference on Control, Automation, Robotics \& Vision, 559-564; DOI: $10.1109 /$ ICARCV.2014.7064365.

Kondili, E., \& Kaldellis, J.K. (2012). Environmental-Social Benefits/Impacts of Wind Power. Comprehensive Renewable Energy, 2, 503-539; DOI: 10.1016/B978-0-08-087872-0.00219-5

Kongkapisuth, K., Roynarin, W., \& Intholo, D. (2017). Study of Building Effects on Small HAWTs Performance in BuildingObstructed Wind Flow Area by Using a CFD k- $\epsilon$ Turbulence Model Validated with Site Measurement. International Energy Journal, 17(4), 193-210.

Lehr, J.H., Keeley, J.W., Kingery, T.B. (Eds.) (2016). Alternative Energy and Shale Gas Encyclopedia. John Wiley \& Sons, New Jersey, USA.

Mahmoud, M.S., \& Xia, Y. (2012). Applied Control System Design. Springer-Verlay London Limited, London, United Kingdom.

Manwell, J.F., McGowan, J.G., \& Rogers, A.L. (2009). Wind Energy Explained, 2nd edn. John Wiley \& Sons Ltd., West Sussex, United Kingdom.

Munson, B.R., Young, D.F., Okiishi, T.H., \& Huebsch, W.W. (2009). Fundamental of Fluid Mechanics, 6th edn. John Wiley \& Sons, Inc, New Jersey, USA.

NI. (2019). Wind Turbine Control Methods. https://www.ni.com/enth/innovations/white-papers/08/wind-turbine-controlmethods.html. Accessed on 24 April 2020.

Ponta, F. L., Otero, A.D., Lago, L.I., \& Rajan, A. (2016). Effects of Rotor Deformation in Wind Turbine Performance: The Dynamic Rotor Deformation Blade Element Momentum Model (DRD-BEM). Renewable Energy, 92, 157-170; DOI: 10.1016/j.renene.2016.01.098

Poradowski, W. (2013). Lecture 7: Turbulence Modeling Introduction to ANSYS Fluent. https://www.academia.edu/36090206/Lecture_7_Turbulence_ Modeling_Introduction_to_ANSYS_Fluent. Accessed on 26 April 2020.

Rao, K.R. (2019). Wind Energy for Power Generation: Meeting the Challenge of Practical Implementation. Springer Nature, Cham, Switzerland.
Rashid, M.H. (2015) Alternative Energy in Power Electronics. Butterworth-Heinemann, Massachusetts, USA.

REN21. Renewables 2018 Global Status Report. https://www.ren21.net/wp-content/uploads/2019/08/FullReport-2018.pdf. Accessed on 12 April 2020.

REN21. Renewables 2019 Global Status Report. https://www.ren21.net/wp-

content/uploads/2019/05/gsr_2019_full_report_en.pdf. Accessed on 12 April 2020.

Rivkin, D.A., Anderson, L.D., \& Silk, L. (2013). Wind Turbine Control Systems. Jones and Bartlett Learning, Massachusetts, USA.

Roynarin, W., Wisatesajja, W., \& Aguila, G. (2020). The Effect of the Number of Blades on the Characteristics of Compressed Air Wind Turbines Using R1235 Airfoil Blade Profile. Proceeding of the $11^{\text {th }}$ International Conference on Mechanical Engineering.

Roynarin, W. (2016). Development of $100 \mathrm{~kW}$ Small Wind Machine for the Floating Applications to Support the Island Energy Demand. Proceedings of the 4th International Conference on Wind Turbine and Renewable Energy.

Simscale. (2020).

K-epsilon. https://www.simscale.com/docs/simulation-setup/globalsettings/k-epsilon/. Accessed on 26 April 2020.

SNC - Lavalin. (2018). Offshore Wind Handbook. https://www.snclavalin.com/ /media/Files/S/SNCLavalin/documents/publications/o-g-offshore-windbrochure_en.pdf. accessed on 14 April 2020.

Society for Underwater Technology. (2018). South West-Offshore Floating Wind-Design and Installation. https://www.sut.org/event/south-west-offshore-floating-winddesign-and-installation/. Accessed on 17 April 2020.

Sodja, J. (2007). Turbulence Models in CFD. Seminar, University of Ljubljana, Slovenia.

Taboada, J.V. (2015). Comparative analysis review on floating offshore wind foundations (FOWF). Proceedings of 54th Naval Engineering and Maritime Industry Congress.

Tey, W.Y., Asako, Y., Sidik, N.A.C., \& Rui-Zher, G. (2017). Governing Equations in Computational Fluid Dynamics: Derivations and a Recent Review. Progress in Energy and Environment, 1, 1-19.

Tong, W. (2010). Fundamentals of Wind Energy. WIT Transactions on State of the Art in Science and Engineering, Volume 44. WIT Press, Southampton, United Kingdom, p. 3-48.

$\mathrm{Tu}$, J., Yeoh, G.H., \& Liu, C. (2018). Computational Fluid Dynamics, 3rd edn. Elsevier Ltd., Amsterdam, Netherlands.

U.S. Department of Energy. Wind Energy Benefits 2015. https://www.nrel.gov/docs/fy 150 osti/62823.pdf. Accessed on 14 April 2020.

Waewsak, J., Landry, M., \& Gagnon, Y. (2015). Offshore Wind Power Potential of the Gulf of Thailand. Renewable Energy, 81, 609-626; DOI: 10.1016/j.renene.2015.03.069 